\title{
Macrocybe titans: The Mushroom Giant of the Western Hemisphere ${ }^{1}$
}

\author{
Elena Karlsen-Ayala and Matthew E. Smith²
}

\section{Summary}

The aptly named Macrocybe titans, meaning "giant head," is the largest known gilled mushroom in the Western Hemisphere. This species was originally described from Florida but can be found widely distributed across the southeastern United States as well as other locations throughout the Caribbean, Central America, and parts of South America. The fruiting bodies (mushrooms) of this fungus are often found in clusters, with the caps growing as large as $100 \mathrm{~cm}$ (3 ft) wide and $30-40 \mathrm{~cm}$ (12-18 inches) tall. Originally described as Tricholoma titans, this mushroom species was first discovered in Gainesville, Florida, in 1973 on submerged wood near a parking lot (Bigelow \& Kimbrough 1980). This mushroom is generally found in disturbed habitats (i.e., near buildings or roads). Over the last 10 years this fungus has been observed in Georgia, Louisiana, North Carolina, South Carolina, and Texas.

\section{Introduction}

Macrocybe titans, originally described as Tricholoma titans, was first described from Florida in 1980 by Howard Bigelow and James Kimbrough. The original collections were found in central and north central Florida. In 1998 this fungus was renamed Macrocybe titans after additional morphological, genetic, and ecological considerations demonstrated that this fungus fit better in the new genus Macrocybe. The genus Macrocybe includes six other subtropical and tropical fungi that are all saprobic (decay) fungi like Macrocybe titans (in contrast, mushrooms in the genus Tricholoma are mostly restricted to temperate forests and are always associated with the roots of particular trees). The current classification of Macrocybe titans is kingdom Fungi, phylum Basidiomycota, class Agaricomycetes, order Agaricales, family Tricholomataceae, genus Macrocybe, and species titans.

\section{Morphology}

The mushrooms of Macrocybe titans are pale buff to cream in color. They can occur alone or in small clusters of 2-3 mushrooms but sometimes grow in dense clusters of up to 10 fruiting bodies (Figure 1). The cap of this mushroom ranges from $8 \mathrm{~cm}$ up to $100 \mathrm{~cm}$ (Figure 1). The mushroom caps are pale buff or cream colored and usually start off convex when young, becoming more flattened with age. Often the edges of the cap are inrolled or scalloped in appearance (Figure 1). The stipe (stem) of $M$. titans ranges 7-20 $\mathrm{cm}$ and is the same color as the cap. The stipe is fibrous and usually scaly (Figure 1) (Bigelow and Kimbrough 1980), and there is no ring around it. The lamellae (gills) are white to pale brown, sinuate (wavy or notched), and crowded (Kimbrough 2000). Spore prints (which can be obtained by placing the cap of the mushroom on a piece of paper or aluminum foil and leaving a jar on top overnight to retain moisture) show that this species has creamy-white-colored spores. The odor of $M$. titans is generally described as mild and nondescript.

1. This document is PP356, one of a series of the Plant Pathology Department, UF/IFAS Extension. Original publication date February 2020. Visit the EDIS website at https://edis.ifas.ufl.edu for the currently supported version of this publication.

2. Elena Karlsen-Ayala, graduate student; and Matthew E. Smith, associate professor, Plant Pathology Department; UF/IFAS Extension, Gainesville, FL 32611.

The Institute of Food and Agricultural Sciences (IFAS) is an Equal Opportunity Institution authorized to provide research, educational information and other services

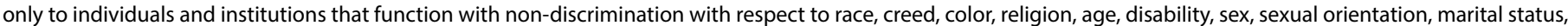

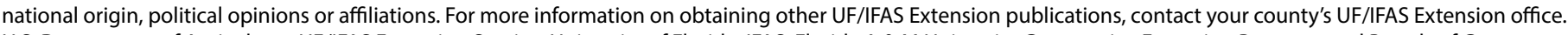
U.S. Department of Agriculture, UF/IFAS Extension Service, University of Florida, IFAS, Florida A \& M University Cooperative Extension Program, and Boards of County Commissioners Cooperating. Nick T. Place, dean for UF/IFAS Extension. 


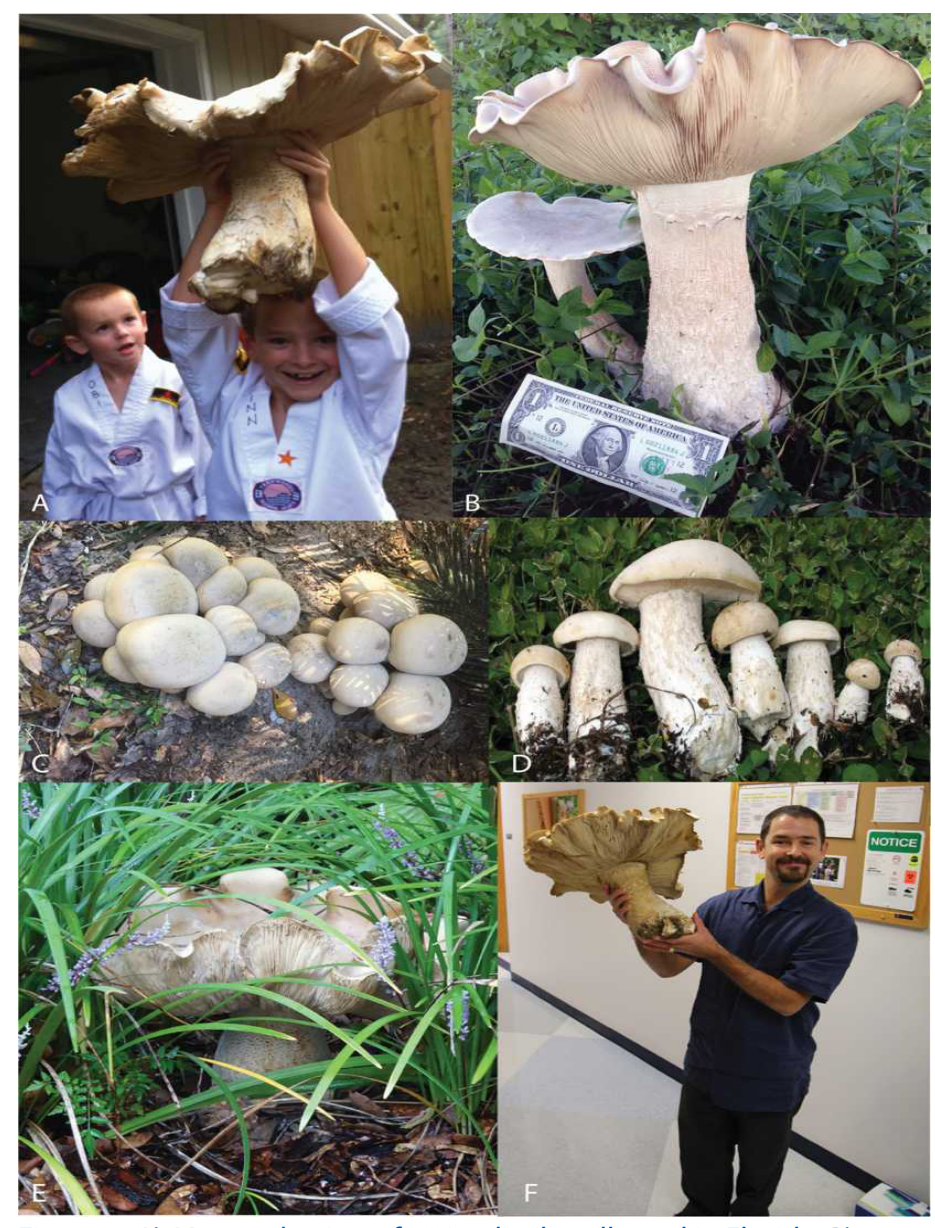

Figure 1. A) Macrocybe titans fruiting body collected in Florida. B) Scalloped, inrolled cap edges and scaly stipe that are common features of $M$. titans. C) Cluster of $M$. titans fruiting bodies from Gainesville, Florida. D) Age series showing the development of $M$. titans fruiting bodies. E) Macrocybe titans fruiting body with scalloped margins found growing in mulch at the edge of a lawn in Gainesville, Florida. F) Dr. Matthew E. Smith holding a fresh M. titans mushroom. Credits: A) Used with permission of Matthew E. Smith, Ph.D.; B) Smith Lab file; C) Jeff B. Davis; D) Richard Kneal (Creative Commons); E) Don Stanhope; F) Phil Harmon, UF/IFAS

Analysis of the tissues through microscopy can also help to differentiate this species from other mushrooms. Examination of the gill tissue shows that the gills of Macrocybe titans have numerous, refractive pseudocystidia (sterile cells) (Pegler et al. 1998). Clamp connections (bumpy structures at the septations) are also present on the fungal hyphae (the filaments that constitute the body of the fungus) (Pegler et al. 1998). Microscopy also shows that the spores are broadly ellipsoid (Bigelow and Kimbrough 1980). These features can only be seen with the aid of a light microscope. Additional identification of $M$. titans includes sequencing a region of the mushroom's DNA (DeLong and Brewer 2013). Polymerase chain reaction (PCR) and DNA sequencing of the internal transcribed spacer (ITS) and 28S large subunit (LSU) regions of the ribosomal DNA, two commonly used DNA barcoding regions for fungi, can also be used to identify M. titans (DeLong and Brewer 2013).

\section{Ecology}

Macrocybe titans is a saprobe, which means that the major food source for this fungus is dead and decaying plant material. Fungi are critical drivers of nutrient cycling in the soil because of their ability to break down lignin and other constituents of plants that are difficult for other organisms to digest (Cooke and Rayner 1984). When fungi break down dead plant tissues, the process releases the nutrients and makes them available for plants and animals in the environment. Macrocybe titans can also be cultured in artificial media in the laboratory. This mushroom species has not been reported to cause disease, infection, or rot of living plant material (DeLong and Brewer 2013). Macrocybe titans does not form any known associations with plants (e.g., this species does not seem to form mycorrhizae or live as an endophyte inside of living plants). Mushrooms of this species have been observed attached to buried roots and wood and in sandy soils and grassy sites during the summer and fall (May to November, with most collections between July and September) (Bigelow and Kimbrough 1980; https://mycoportal.org/). This mushroom is often found in disturbed sites near parking lots, roadsides, or buildings, suggesting that it is adapted to disturbance and thrives outside of natural areas (Figure 1).

\section{Range and Habitat}

The genus Macrocybe contains seven described species that are all widely distributed in the tropics. Macrocybe titans is restricted to the neotropics (tropical and subtropical habitats in the Western Hemisphere), but other species have been reported in the Palaeotropics in areas such as Taiwan and India. Macrocybe titans has been collected or reported from across the southern United States, including in Florida, Georgia, Louisiana, North Carolina, South Carolina, and Texas (Figure 2). Records from Central America and the Caribbean include reports from Mexico, Belize, Costa Rica, Panama, Puerto Rico, Trinidad, and the US Virgin Islands. There have been additional reports of $M$. titans from South America, including in Argentina, Brazil, Colombia, Ecuador, and Venezuela (Ramírez et al. 2017).

\section{Range Expansion in the United States}

William A. Murrill (1869-1957) was an avid mycologist who collected and described mushrooms all over Florida and the world in the early 1900s (Weber 1961). He collected over 700,000 specimens and described approximately 700 fungal species in Florida, mostly in north central Florida. Despite the fact that he lived and collected in Gainesville 
for more than 20 years, he never reported or described Macrocybe titans. Given that $M$. titans is the largest known mushroom species in the Americas, it seems likely that W. A. Murrill would have reported this species had he seen it in Florida. We suspect that $M$. titans was either restricted to the tropical zone of southern Florida or more likely was introduced to Florida sometime during the early to mid1900s from other parts of the Caribbean. We also hypothesize that this species has been spreading within the southeastern United States since its introduction. Our hypothesis is based on fruiting records from the Mycoportal (https:// mycoportal.org/). These observations suggest that within the United States, $M$. titans was restricted to Florida during the 1970s and 1980s and has only more recently spread to other states (Figure 2). Macrocybe titans may be naturally dispersing via spores, but we also suspect that the movement of wood mulch and soil may also have facilitated the movement of this fungus from Florida to other US states. Given that $M$. titans is a tropical species, we also suspect that the warming climate (particularly the reduction in the average number of hours below freezing each year) may facilitate the range expansion of this mushroom.

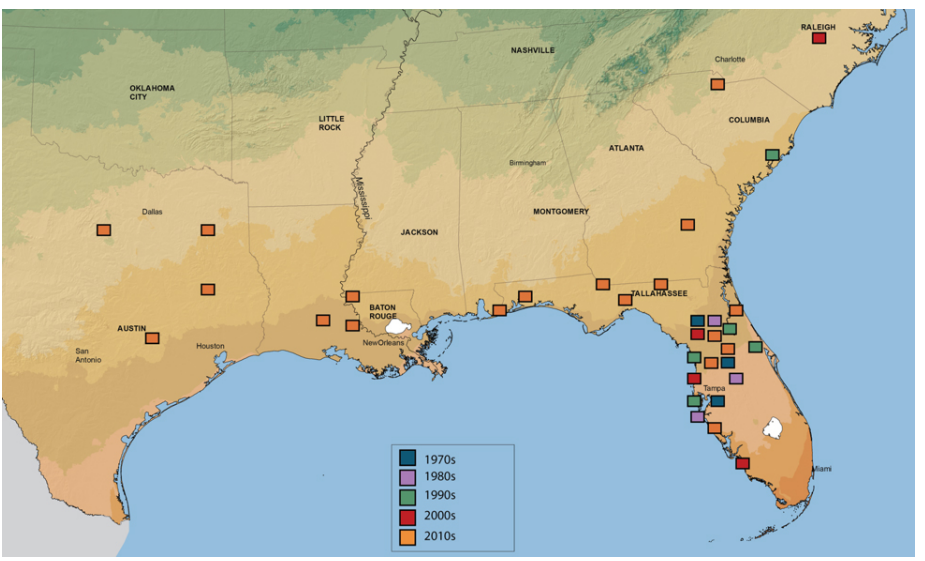

Figure 2. North American distribution of Macrocybe titans based on specimen records in the Mycoportal (https://mycoportal.org/). Squares represent locations where the mushroom species has been collected in the continental United States. The map was adapted from USDA Plant Hardiness Zones; differences in map colors represent differences in mean extreme minimum temperatures.

\section{Names}

Current name: Macrocybe titans (1998)

Original name: Tricholoma titans (1980)

\section{References}

Bessette, A. E., W. C. Roody, A. R. Bessette, and D. L. Dunaway. 2007. Mushrooms of the Southeastern United States. Syracuse, NY: Syracuse University Press.

Bigelow, H., and J. Kimbrough. 1980. "Tricholoma titans, a New Species from Florida.” Mycotaxon 11 (2): 425-429.

Cooke, R. C., and A. D. Rayner. 1984. Ecology of Saprotrophic Fungi. London: Pearson Longman.

DeLong, J., and M. T. Brewer. 2019. Macrocybe titans: Largest Mushroom Species in the Western Hemisphere Found Growing in Georgia. Circular 1033. Athens, GA: University of Georgia Extension.

Kimbrough, J. W. 2000. Common Florida Mushrooms. SP 256. Gainesville: University of Florida Institute of Food and Agricultural Sciences.

"MyCoPortal: The Mycology Collections Data Portal." 2020. http://mycoportal.org/portal/index.php. Accessed 8 January 2020.

Pegler, D. N., D. J. Lodge, and K. K. Nakasone. 1998. “The Pantropical Genus Macrocybe gen. nov." Mycologia 90 (3): 494-504. https://doi.org/10.1080/00275514.1998.12026934

Ramírez, N. A., N. Niveiro, S. A. Michlig, and O. F. Popoff. 2017. "First Record of Macrocybe titans (Tricholomataceae, Basidiomycota) in Argentina." Check List 13 (4): 153-158. http://dx.doi.org/10.15560/13.4.153

Weber, G. 1961. "William Alphonso Murrill.” Mycologia 53 (6): 543-557. https://doi.org/10.1080/00275514.1961.1201 7984

\section{Edibility}

Macrocybe titans has been reported by some authors to be edible (Kimbrough 2000; Bessette et al. 2007). However, because $M$. titans could be confused during young stages with common poisonous species (such as Chlorophyllum molybdites), members of the public should never consume wild mushrooms that they cannot positively identify as edible. When in doubt, throw it out! 